\title{
Intérêt d'une sélection associant valeur en fourrage et valeur en grain pour l'amélioration du maïs fourrage et du maïs grain
}

\author{
J.P. Sampoux, A. Gallais et M. Lefort-Buson \\ INRA-CNRS-UPS, ferme du Moulon, 91190 Gif sur Yvette, France
}

(reçu le 13 juin 1988, accepté le 20 avril 1989)

Résumé - Les descendances en croisement avec un testeur hybride simple de 4 populations de maïs précoce ont été récoltées en plante entière (stade ensilage) et en grain. La corrélation génétique entre le rendement en plante entière et le rendement en grain varie entre 0,71 et 0,88 , selon les populations. La corrélation génétique entre les caractères de précocité de récolte fourrage et grain (teneur en matière sèche de la plante entière et humidité du grain) est assez variable d'une population à l'autre. Dans le cas d'un objectif de sélection fourrage précoce, une sélection indirecte sur les performances grain conduit à une réponse corrélative attendue sur le rendement en plante entière inférieure de $35 \%$ en moyenne au progrès attendu par une sélection directe fourrage. Une sélection exclusivement fourrage entraîne, quant à elle, une réponse corrélative attendue sur le rendement en grain inférieure de $20 \%$ en moyenne à la réponse à la sélection grain. Ces résultats montrent donc la nécessité de différencier la sélection fourrage de la sélection grain dès le début des programmes d'amélioration. Une double récolte en plante entière et en grain peut toutefois être utile pour maintenir une proportion minimale de grain en sélection fourrage. Elle peut également être nécessaire en sélection grain pour éviter une perte de variabilité trop rapide sur la biomasse aérienne totale. L'efficacité de la sélection peut alors être renforcée de façon importante par une estimation multicaractère des critères de sélection, combinant l'information apportée par les 2 types de récolte.

Zea mays - sélection multicaractère - index de sélection - valeur en fourrage - indice de récolte grain

Summary - Combining forage and grain tests for both forage and grain maize breeding. Topcross progenies with a single cross tester from 4 early maize populations were tested for whole-plant yield (silage maturity) and for grain yield. Genetic correlation between whole plant yield and grain yield varies with populations from 0.71 to 0.88 . Genetic correlations between earliness criteria of forage yield (dry matter content of whole plant) and grain yield (grain moisture) were quite different in the 4 populations. For an early forage breeding aim, an indirect selection upon grain tests led to an expected response on forage yield $35 \%$ lower on average, than direct forage selection. A selection upon forage tests led to an average of $20 \%$ less than the expected response on grain yield than grain selection. So, evidence suggests that forage selection has to be separated from grain selection from the beginning of breeding programmes. However, testing for both whole-plant yield and grain yield may be useful to keep a minimum grain content in forage selection. It can also be useful in grain selection to avoid a too fast decrease of whole-plant variability. In such cases, multicharacter estimation of breeding criteria combining both forage and grain traits can increase selection efficiency.

Zea mays - multitrait selection - selection index - forage value - harvest index

\section{INTRODUCTION}

L'intérêt d'une sélection de maïs précoces, destinés à un ensilage en plante entière, différente de la sélection grain a déjà été clairement démontré. Différents travaux ont en effet prouvé qu'un développement important de la partie non-grain était nécessaire pour obtenir un rendement en plante entière élevé (Gallais et al., 1983; Geiger et al.,
1986). La partie non-grain semble, d'autre part, présenter une variabilité génétique qui pourrait être utilisée pour l'amélioration de la valeur alimentaire (Gallais et al., 1981; Deinum \& Struik, 1986) et de la précocité de dessèchement (Geiger et al., 1986) de la plante entière.

Le maintien d'une proportion de grain suffisamment importante dans lensilage est cependant nécessaire pour : 
- maintenir une activité photosynthétique assez importante après la floraison, par effet de "puits» de la partie grain (Daynard \& Tollenaar, 1984),

- assurer une teneur en matière sèche suffisante à la récolte (Gallais et al., 1981; Hunter, 1986),

- obtenir un bon niveau de valeur alimentaire.

Sur ce dernier point, Lila et al., (1986) constatent en effet, avec 2 méthodes de digestibilité in vitro, une interaction génotype $x$ milieu pour la digestibilité de la partie non-grain , estompée au niveau de la plante entière grâce à une proportion de grain assez élevée. Les interactions entre proportion de grain et valeur alimentaire de la partie non-grain restent cependant à clarifier. La proportion minimale de grain à retenir en sélection fourrage est sans doute à définir en fonction de la zone de précocité et du type d'élevage visés, ainsi que du matériel génétique utilisé.

En ce qui concerne l'amélioration des performances grain pour les types demi-précoces et précoces, Barrière et al. (1987) montrent que le progrès génétique acquis entre 1950 et 1980 provient principalement d'une augmentation de l'indice de récolte pour les variétés demi-précoces, et que la réponse corrélative de la biomasse aérienne totale ne dépasse pas le progrès acquis en grain pour les variétés précoces. Une sélection directe sur la biomasse aérienne totale pourrait donc être nécessaire pour obtenir de nouveaux progrès génétiques importants sur le rendement en grain.

L'élimination de génotypes peu productifs en grain, dans le cadre d'une sélection pour la valeur en fourrage, peut se réaliser de façon simple sur un grand nombre de génotypes, par une double récolte, en plante entière et en grain, sur des essais différents. Un tel protocole expérimental peut également être envisagé en sélection grain pour maintenir un progrès génétique suffisant sur le rendement en biomasse aérienne totale. L'intérêt d'une double expérimentation, en plante entière et en grain, est alors à discuter par rapport à une expérimentation exclusivement consacrée au mode de récolte visé par l'objectif de sélection. Cette étude a donc pour but de préciser, sur différentes populations, l'intérêt et les modalités d'une sélection récurrente combinant les caractères observés à la récolte en fourrage et à la récolte en grain, pour chacun des 2 objectifs de sélection, fourrage et grain. Elle permettra de plus de vérifier l'intérêt à différencier, à partir d'un même matériel, la sélection fourrage de la sélection grain. Ces différents aspects ne seront abordés que pour les critères de productivité et de précocité en récolte fourrage et grain.

\section{MATÉRIEL ET MÉTHODES}

\section{Matériel et méthode de sélection}

Les résultats présentés ont été obtenus sur les test de descendances d'un premier cycle de sélection récurrente de 4 populations de maïs précoces (Tableau I), déjà étudiées par ailleurs (Sampoux et al., 1989). Pour toutes les populations étudiées, un cycle de 3 ans a été choisi. En première année, les plantes $S_{0}$ de chaque population ont été autofécondées et croisées avec le testeur. En deuxième année, les descendances en croisement des plantes $S_{0}$ ont été installées en essais récoltés en fourrage. Suivant les populations, une partie ou l'ensemble des descendances en croisement a également fait l'objet d'une expérimentation récoltée en grain (Tableau II). En troisième année, les descendances $S_{1}$ des piantes $S_{0}$ sélectionnées ont été intercroisées pour produire les populations du cycle de sélection suivant.

Les tests de seconde année ont été réalisés à la Ferme du Moulon, en 1984 pour la population SRC84, et en 1985 pour les populations SRC85, SRD85, et SRCD85. Le dispositif expérimental de chaque population, composé de plusieurs lattices à 2 répétitions, interconnectés par les variétés témoins Brulouis (précoce) et Dea (demi-précoce), est reporté dans le Tableau II avec les effectifs testés. Les parcelles étaient formées d'une ligne de $5 \mathrm{~m}$ et espacées de $0,80 \mathrm{~m}$. Les densités de peuplement retenues étaient de 95000 plantes/ha pour les essais fourrage et de 85000 plantes/ha pour les essais grain.

Tableau I. Populations étudiées et leurs testeurs.

$\begin{array}{llll}\text { Dénomination } & \begin{array}{c}\text { Population } \\ \text { Groupe de combinaison des lignées } \\ \text { constitutives }\end{array} & \text { Formule } & \text { Testeur } \\ & & & \\ & \text { cornées } & \text { F250 F252 } & \text { denté } \\ \text { SRC85 } & \text { dentées } & \text { F2 F251 } & \text { corné } \\ \text { SRD85 } & \text { cornées et dentées } & \text { F251 F252 } & \text { corné-denté } \\ \text { SRCD85 } & \text { cornées } & \text { F250 F252 } & \text { denté }\end{array}$


Tableau II. Protocoles expérimentaux.

\begin{tabular}{|c|c|c|c|c|c|c|}
\hline \multirow[t]{3}{*}{ Population } & \multicolumn{6}{|c|}{ Descendances en croisement } \\
\hline & \multicolumn{3}{|c|}{ Récoltées en fourrage } & \multicolumn{3}{|c|}{ Récoltées en grain } \\
\hline & $\begin{array}{c}\text { Nombre de } \\
\text { descendances }\end{array}$ & $\begin{array}{l}\text { Nombre } \\
\text { d'essais }\end{array}$ & $\begin{array}{l}\text { Dispositif } \\
\text { lattice }\end{array}$ & $\begin{array}{c}\text { Nombre de } \\
\text { descendances }\end{array}$ & $\begin{array}{l}\text { Nombre } \\
\text { d'essais }\end{array}$ & $\begin{array}{c}\text { Dispositif } \\
\text { lattice }\end{array}$ \\
\hline SRC85 & 156 & 3 & $7^{*} 8$ & 104 & 2 & $7^{\star} 8$ \\
\hline SRD85 & 156 & 3 & $7^{\star} 8$ & 104 & 2 & $7 * 8$ \\
\hline SRCD85 & 208 & 4 & $7^{*} 8$ & 208 & 4 & $7 * 8$ \\
\hline SRC84 & 204 & 3 & $8^{*} 9$ & 77 & 1 & $9 * 9$ \\
\hline
\end{tabular}

2 répétitions pour tous les essais.

\section{Caractères observés}

Différents caractères ont été observés en cours de végétation. La date de floraison femeile (dflo) correspond à la date à laquelle $50 \%$ des soies sont visibles. Certains caractères de morphologie ont été observés après la floraison sur un échantillon de 5 plantes par parcelle: la hauteur totale (ht), du sol à la base de la première ramification de la panicule, la hauteur de l'épi supérieur ((he), du sol au nœud de l'épi, la longueur de la feuille de l'épi supérieur (long) et sa largeur (larg), mesurée dans la zone la plus large.

Les essais plante entière d'une population, groupés, sont récoltés en une même journée pour un niveau de teneur en matière sèche compris entre 25 et $30 \%$, de façon à observer des différences de teneur en matière sèche entre génotypes liées à la précocité, et indépendantes de phénomènes de senescences aléatoires pouvant survenir à un stade plus avancé (Barloy, 1984). Pour chaque parcelle, la matière verte totale est pesée. Le rendement sec en plante entière ( $r d t f$ ) est calculé après estimation de la teneur en matière sèche (\%ms f) par séchage à l'étuve d'un échantillon de matière verte de $800 \mathrm{~g}$.

Les essais grain d'une population sont également récoltés en une même journée pour un niveau moyen d'humidité du grain (30 à 35\%) permettant une récolte mécanique correcte. Le rendement sec en grain (rdt g) de chaque parcelle est calculé à partir du poids de grain humide recueilli et d'une estimation de l'humidité du grain, réalisée par lecture directe à l'humidimètre en 1984, et par sèchage à l'étuve d'un échantillon de $200 \mathrm{~g}$ en 1985 . Le rendement $\mathrm{sec}$ en grain observé à ce stade peut permettre d'éliminer les génotypes à faible proportion de grain à la récolte du fourrage. Au stade $30 \%$ de matière sèche de la plante entière, le poids sec de la partie grain peut en effet être estimé à au moins $70 \%$ de sa valeur finale, avec les types de maïs actuels (Daynard et Hunter, 1975; Barloy, 1984).

Des notations de sensibilité à la verse ont été réalisées en cours de végétation et à la récolte des essais fourrage et grain. Des notations de pourriture de tiges ont également été effectuées sur les essais grain. Elles n'ont pas été retenues dans cette étude, car elles conduisent souvent à des variances résiduelles importantes. Une sélection par niveaux indépendants, à partir des notations de verse et d'un index des autres caractères, semble un bon compromis tant que de meilleurs critères de sensibilité à la verse n'auront pas été définis.

\section{Interprétation biométrique des test de secon- de année}

L'analyse de la variance et l'estimation des composantes génétiques et résiduelles de la variance phénotypique ont déjà été décrites (Sampoux et al., 1989). Les covariances résiduelles entre caractères observés sur l'expérimentation grain et caractères observés sur l'expérimentation fourrage étant, par construction, d'espérance nulle, les covariances phénotypiques entre ces caractères sont égales aux covariances génétiques.

La meilleure estimation de la valeur génétique d'un caractère, tenant compte des relations entre différents caractères observés, est obtenue par régression génophénotypique (Henderson, 1975; Vincourt \& Gallais, 1983). Le degré de précision de l'estimation de la valeur génétique de certains caractères est estimé par le carré de la corrélation entre valeur génétique vraie et valeur estimée $\left[r^{2}(G, \hat{G})\right]$, pour une sélection univariable (héritabilité univariable), comme pour l'observation de plusieurs caractères (héritabilité multivariable). Les coefficients $r^{2}(G, \hat{G})$ seront également calculés pour le cas théorique de 4 répétitions, ce calcul étant réalisé à partir des matrices de variance-covariance génétique et résiduelle, estimées sur 2 répétitions. Différents index des estimateurs des caractères principaux seront construits. Les pondérations économiques seront déterminées en fonction des progrès génétiques souhaités (Rouvier, 1977). En l'absence d'épistasie, l'estimation de la différence entre la moyenne des familles sélectionnées sur index et la moyenne générale de la population est également une estimation du progrès génétique attendu après intercroisement. Les progrès génétiques seront estimés pour une intensité de sélection de $20 \%$.

\section{RÉSULTATS}

\section{Coefficients de variation génétique et rési- duelle, héritabilités univariables}

La comparaison des ordres de grandeur des coefficients de variation génétique (écart-type génétique rapporté à la moyenne) (Tableau III) fait ressortir une variabilité génétique un peu plus importante pour le rendement en grain que pour le rendement en fourrage, dans 3 populations sur 
Tableau III. Coefficients de variation et héritabilités univariables observées sur les descendances en croisement des populations SRC85 (a), SRD85 (b), SRCD85 (c), SRC84 (d).

\begin{tabular}{|c|c|c|c|c|c|c|c|c|}
\hline \multirow[b]{2}{*}{$\begin{array}{l}\text { coefficient de varia- } \\
\text { tion génétique }\end{array}$} & \multicolumn{2}{|c|}{$\begin{array}{l}\text { Rendement fourrage } \\
\text { rdt } f\end{array}$} & \multicolumn{2}{|c|}{$\begin{array}{l}\% m s \\
\% m s f\end{array}$} & \multicolumn{2}{|c|}{$\begin{array}{l}\text { Rendement grain } \\
\qquad d t g\end{array}$} & \multicolumn{2}{|c|}{$\begin{array}{l}\text { Humidité du grain } \\
\text { hum } g\end{array}$} \\
\hline & $\begin{array}{l}5,4(a) \\
6,3(b)\end{array}$ & $\begin{array}{l}4,9(c) \\
5,1(d)\end{array}$ & $\begin{array}{l}3,9 \\
4,0\end{array}$ & $\begin{array}{l}3,4 \\
3,3\end{array}$ & $\begin{array}{l}9,1 \\
6,8\end{array}$ & $\begin{array}{l}6,4 \\
7,8\end{array}$ & $\begin{array}{l}5,2 \\
5,9\end{array}$ & $\begin{array}{l}4,7 \\
3,8\end{array}$ \\
\hline $\begin{array}{l}\text { coefficient de varia- } \\
\text { tion résiduelle }\end{array}$ & $\begin{array}{l}8,8 \\
7,7\end{array}$ & $\begin{array}{l}6,3 \\
8,7\end{array}$ & $\begin{array}{l}3,5 \\
3,2\end{array}$ & $\begin{array}{l}3,8 \\
5,4\end{array}$ & $\begin{array}{l}8,2 \\
8,5\end{array}$ & $\begin{array}{r}9,3 \\
12,9\end{array}$ & $\begin{array}{l}4,2 \\
3,3\end{array}$ & $\begin{array}{l}4,7 \\
2,9\end{array}$ \\
\hline $\begin{array}{l}\text { Héritabilité univa- } \\
\text { riable (intervalle de } \\
\text { confiance à } 5 \% \text { ) }\end{array}$ & $\begin{array}{c}0,44 \\
(0,21-0,60) \\
0,53 \\
(0,35-0,66)\end{array}$ & $\begin{array}{c}0,56 \\
(0,41-0,66) \\
0,38 \\
(0,18-0,53)\end{array}$ & $\begin{array}{c}0,70 \\
(0,58-0,79) \\
0,74 \\
(0,64-0,81)\end{array}$ & $\begin{array}{c}0,65 \\
(0,53-0,73) \\
0,44 \\
(0,26-0,58)\end{array}$ & $\begin{array}{c}0,71 \\
(0,57-0,80) \\
0,56 \\
(0,35-0,70)\end{array}$ & $\begin{array}{c}0,49 \\
(0,33-0,62) \\
0,43 \\
(0,08-0,64)\end{array}$ & $\begin{array}{c}0,74 \\
(0,62-0,82) \\
0,86 \\
(0,80-0,91)\end{array}$ & $\begin{array}{c}0,68 \\
(0,57-0,75) \\
0,77 \\
(0,64-0,86)\end{array}$ \\
\hline
\end{tabular}

4. Les héritabilités univariables du rendement en grain ne sont toutefois pas significativement (au seuil $5 \%$ ) plus élevées que celles du rendement en fourrage.

Dans quelques cas, les caractères de précocité de récolte (teneur en matière sèche du fourrage et humidité du grain) présentent une héritabilité univariable (Tableau III) significativement plus élevée (au seuil $5 \%$ ) que le critère de rendement correspondant (fourrage ou grain).

\section{Corrélations génétiques}

La corrélation génétique entre rendement en grain et rendement en fourrage (Tableau IV) apparaît assez élevée pour toutes les populations étudiées (0.71 à 0.88$)$. La corrélation entre la teneur en matière sèche du fourrage et l'humidité du grain est très variable selon les populations, même au sein d'une année donnée (Tableau IV). En fourrage comme en grain, les corrélations génétiques entre critère de rendement et critère de précocité de récolte (Tableau IV) sont assez variables selon les populations. Cependant, les corrélations fortes traduisent toujours une opposition entre productivité et précocité de récolte; cette opposition n'est observée à la fois en fourrage et en grain que dans une population (SRC85).

\section{Sélection sur un seul mode de récolte des descendances en croisement}

\section{Sélection sur les performances fourrage}

Pour chaque population, une estimation multicaractère du rendement en fourrage et de la teneur en matière sèche a été réalisée, en prenant en compte les données recueillies à la récolte du fourrage et l'information apportée par les caractères observés en cours de végétation. Une telle pratique conduit en effet à une estimation plus précise de la valeur génétique du rendement en fourrage (Tableau $V$ ) pour toutes les populations étudiées (Sampoux et al., 1989). Un index des valeurs génétiques estimées du rendemient en fourrage et de la teneur en matière sèche a été construit. Les pondérations de l'index de chaque population (Tableau VI) ont été établies de façon à obtenir le progrès génétique le plus élevé possible sur le rendement en fourrage, sans évolution du niveau de précocité de récolte (\%ms $f$ ). La teneur en matière sèche moyenne de chaque population est, en effet, comprise entre la valeur

Tableau IV. Corrélations génétiques entre caractères observés à la récolte des essais fourrage et grain des descendances en croisement.

\begin{tabular}{lrrr}
\hline & \multicolumn{1}{c}{$r d t f$} & $\% m s f$ & \multicolumn{1}{c}{$r d t g$} \\
\hline \multirow{4}{*}{ humg } & (a) 0,71 & $-0,48$ & $0,55(0,13)$ \\
& (b) 0,22 & $-0,59$ & $0,50(0,14)$ \\
& (c) 0,09 & $-0,71$ & $0,22(0,13)$ \\
& (d) 0,08 & $-0,23$ & $-0,24(0,20)$ \\
rdtg & & & \\
& 0,80 & $-0,18$ & \\
& 0,88 & $-0,07$ & \\
& 0,71 & $-0,24$ & \\
& 0,81 & 0,52 & \\
& & & \\
& $-0,43(0,19)$ & & \\
& $0,18(0,13)$ & & \\
& $-0,22(0,14)$ & & \\
& $-0,52(0,26)$ & &
\end{tabular}

Populations: SRC85 (a), SRD85 (b), SRCD85 (c), SRC84 (d) Entre parenthèses: erreur standard (Scheinberg, 1966), estimable uniquement quand les 2 caractères sont observés sur le même essai. 
Tableau V. Précision de l'estimation de la valeur génétique $\left[r^{2}(G, G)\right]$ des critères de rendement et de précocité de récolte fourrage et grain différents pour protocoles d'expérimentation.

\begin{tabular}{|c|c|c|c|c|c|c|c|c|c|c|}
\hline \multicolumn{2}{|c|}{ Protocole expérimental } & \multirow{3}{*}{$\begin{array}{l}\text { Caractères } \\
\text { prédicteurs }\end{array}$} & \multicolumn{8}{|c|}{$r^{2}(G, \hat{G})$} \\
\hline & & & \multicolumn{2}{|c|}{$r d t f$} & \multicolumn{2}{|c|}{$\% m s f$} & \multicolumn{2}{|c|}{$r d t g$} & \multicolumn{2}{|c|}{ hum $g$} \\
\hline \multirow{5}{*}{$\begin{array}{l}2 \text { répétitions } \\
\text { des } \\
\text { descendances } \\
\text { en croisement }\end{array}$} & sélection univariable directe & & $0,44(a)$ & $0,56(c)$ & 0,70 & 0,65 & 0,71 & 0,49 & 0,74 & 0,68 \\
\hline & \multirow{2}{*}{2 répétitions fourrage } & \multirow{2}{*}{ A } & & & & & & & & \\
\hline & & & 0,60 & 0,59 & $\begin{array}{l}0,84 \\
0,76\end{array}$ & $\begin{array}{l}0,71 \\
0,6 i\end{array}$ & & & & \\
\hline & \multirow[t]{2}{*}{2 répétitions grain } & \multirow[t]{2}{*}{$\mathrm{B}$} & & & & & 0,77 & 0,58 & 0,79 & 0,70 \\
\hline & & & & & & & 0,74 & 0,48 & 0,89 & 0,79 \\
\hline \multirow{6}{*}{$\begin{array}{l}4 \text { répétitions } \\
\text { des } \\
\text { descendances } \\
\text { en croisement }\end{array}$} & \multirow{2}{*}{4 répétitions fourrage } & \multirow[t]{2}{*}{$A_{0}$} & 0,68 & 0,74 & 0,87 & 0,81 & & & & \\
\hline & & & 0,70 & 0,66 & 0,86 & 0,71 & & & & \\
\hline & \multirow[t]{2}{*}{4 répétitions grain } & \multirow[t]{2}{*}{$\mathrm{B}_{0}$} & & & & & 0,85 & 0,67 & 0,87 & 0,81 \\
\hline & & & & & & & 0,75 & 0,60 & 0,93 & 0,87 \\
\hline & \multirow{2}{*}{$\begin{array}{l}2 \text { répétitions fourrage } \\
\text { et } 2 \text { répétitions grain }\end{array}$} & \multirow[t]{2}{*}{ C } & 0,75 & 0,66 & 0,79 & 0,74 & 0,77 & 0,60 & 0,80 & 0,73 \\
\hline & & & 0,66 & 0,73 & 0,78 & 0,69 & 0,74 & 0,57 & 0,89 & 0,78 \\
\hline
\end{tabular}

$A_{0}: r d t, \% m s f$

$\mathrm{A}: \mathrm{A}_{0}, h t$, he, long, dflo

$\mathrm{B}_{0}:$ rdt g, hum $g$

$\mathrm{B}: \mathrm{B}_{0}, h t, h e$, long, dflo

$\mathrm{C}: \mathrm{A}_{0} \cdot \mathrm{B}_{0}$

Populations: SRC85 (a), SRD85 (b), SRCD85 (c), SRC84 (d)

Tableau VI. Sélection sur index. Coefficients appliqués et progrès génétiques attendus $\left({ }_{1} \Delta_{\mathrm{g}}\right)$.

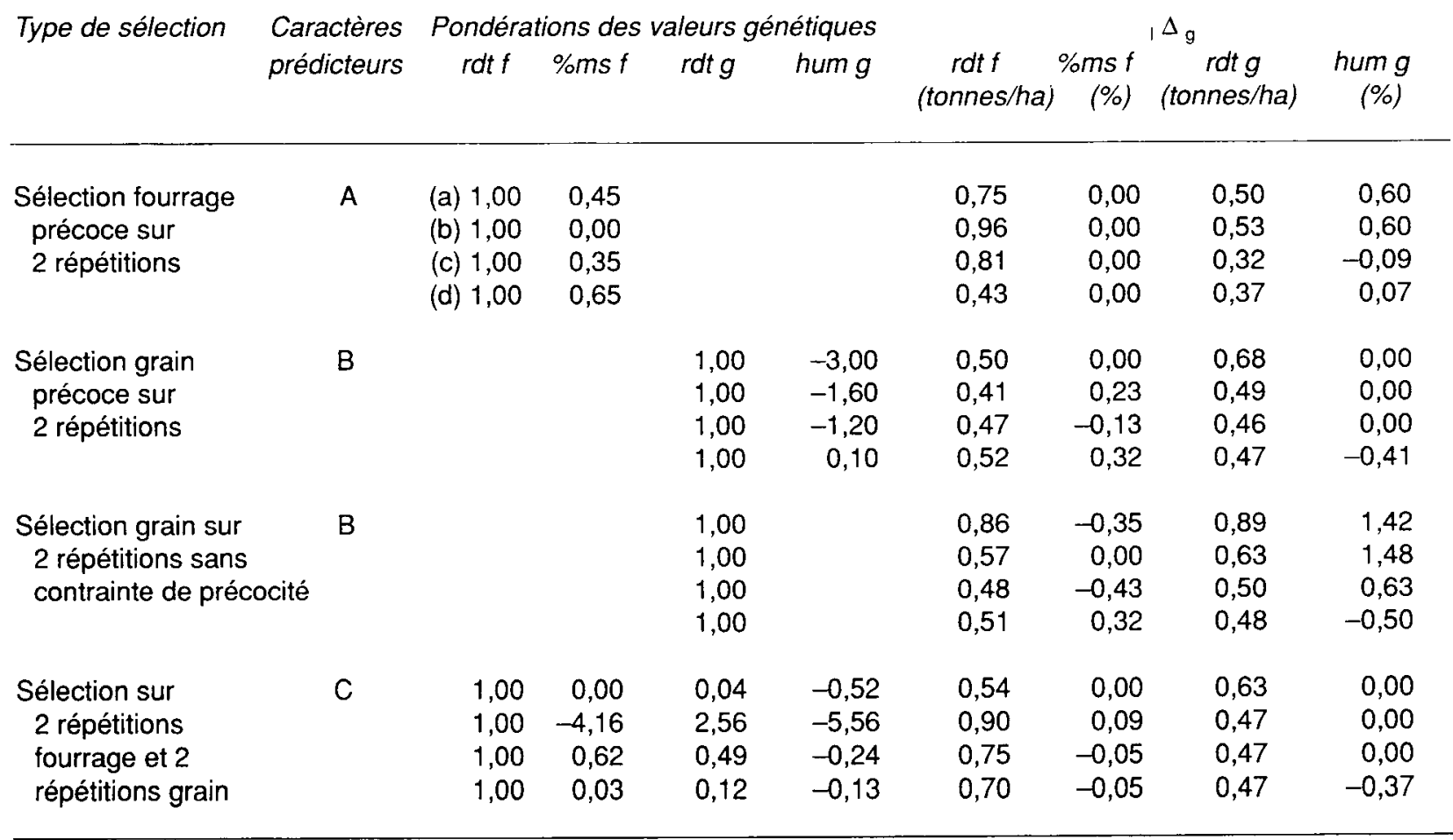

\footnotetext{
A: rdt $f, \% m s f, h t$, he, long, dflo

B: rdt g, hum $g$, ht, he, long, dflo

C: rdt $f, \% m s f$, rdt $g$, hum $g$
}

Populations: SRC85 (a), SRD85 (b), SRCD85 (c), SRC84 (d) 
du témoin précoce Brulouis et celle du témoin demi-précoce Dea (Tableau VII), ce qui semble être un niveau de précocité acceptable pour un objectif de sélection fourrage précoce.

Le progrès génétique attendu sur le rendement en fourrage reste moins important pour la population SRC84 que pour les autres populations (Tableau VI), du fait, d'une part, de la corrélation négative liant ce caractère à la teneur en matière sèche, et, d'autre part, de son héritabilité multivariable plus faible que dans les autres populations. La réponse corrélative du rendement en grain est équivalente au progrès génétique attendu par sélection grain pour la population SRD85, mais inférieure à celui-ci (de 25 à $30 \%$ ) pour les autres populations (Tableau VI). La réponse corrélative de l'humidité du grain (Tableau VI) laisse prévoir une perte de précocité en récolte grain pour 3 populations.

\section{Sélection sur les performances grain}

L'information apportée par les caractères observés en cours de végétation a également été prise en considération pour l'estimation du rendement et de l'humidité du grain. Cette information apporte cependant un gain de précision moins important sur le rendement en grain que sur le rendement en fourrage (Tableau V). L'index de sélection est une combinaison linéaire des valeurs génétiques estimées du rendement en grain et de l'humidité de grain.

\section{Sélection pour un objectif grain précoce}

Pour cet objectif de sélection, le niveau recherché de précocité des populations correspond à une humidité moyenne du grain comprise entre celle du témoin précoce et celle du témoin demiprécoce. Pour les populations expérimentées en 1985, les pondérations de l'index ont donc été choisies de façon à obtenir un maintien du niveau de précocité de récolte (hum g); un gain de précocité a, par contre, semblé nécessaire pour la population SRC84 (Tableaux VI et VII).

La réponse corrélative attendue sur le rendement en fourrage est de l'ordre de grandeur de la réponse directe attendue sur le rendement en grain, quelle que soit la population (Tableau VI). Pour les populations expérimentées en 1985, la réponse corrélative du rendement en fourrage reste cependant nettement inférieure (de $30 \%$ à plus de $50 \%$ ) au progrès attendu sur ce caractère par sélection sur performances fourrage (Tableau VI). Dans le cas de la population SRC84, la sélection grain est par contre plus efficace que la sélection fourrage pour améliorer le rendement en fourrage (Tableau VI), cela étant en partie lié à la faible héritabilité de ce caractère sur l'expérimentation fourrage.

\section{Sélection grain sans contrainte sur la précocité de récolte}

Le poids accordé à la précocité de récolte grain dans la sélection grain décrite ci-dessus peut toutefois être jugé trop important pour un objectif de sélection fourrage. Un autre index de sélection sur performances grain a donc été construit, en n'imposant aucune contrainte sur l'humidité du grain; il permet ainsi d'atteindre le progrès génétique maximal sur le rendement en grain.

La sélection sur un tel index conduit à une réponse corrélative attendue sur le rendement en plante entière (Tableau VI):

- toujours nettement inférieure au progrès attendu par sélection directe sur performances fourrage pour les populations SRD85 et SRCD85,

- mais supérieure à celui-ci pour les populations cornées SRC84 et SRC85. Ces réponses corrélatives assez fortes obtenues pour les populations cornées peuvent être imputées à une héri-

Tableau VII. Moyennes des témoins et des descendances en croisement des populations SRC85 (a), SRD85 (b), SRCD85 (c), SRC84 (d).

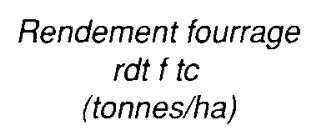
Rendement fourrage
(tonnes/ha)

(20)


tabilité du rendement en fourrage plus faible que celle du rendement en grain (Tableau III). Cependant, ce second type de sélection grain conduit à une perte de précocité en récolte fourrage pour la population SRC85 (mais également pour la population SRCD85), cela n'est donc pas une méthode de sélection efficace pour un objectif fourrage précoce.

\section{Sélection combinant les performances four- rage et grain des descendances en croise- ment}

\section{Recherche d'une meilleure efficacité de la sélection sur les critères fourrage}

\section{Rendement en fourrage}

Pour augmenter la précision de l'estimation de la valeur génétique du rendement en fourrage des populations SRC85 et SRD85 (Tableau V), une récolte en grain peut compléter la récolte en fourrage de façon aussi efficace que l'observation de caractères en cours de végétation sur l'expérimentation fourrage. La récolte en grain est même nettement plus efficace que l'observation des caractères de morphologie, dans le cas de la population SRC84; le gain de précision obtenu est alors équivalent à celui fourni par les observations réalisées sur descendances $S_{1}$ (Sampoux et al., 1989). Pour les populations SRC85 et SRC84, une récolte en fourrage et en grain est plus efficace qu'un doublement du nombre de répétitions récoltées en fourrage (Tableau $\mathrm{V}$ ). Pour la population SRC85, la récolte en grain apporte également un gain de précision sur le rendement en fourrage, inférieur cependant à celui procuré par l'observation de caractères en cours de végétation (Tableau $\mathrm{V}$ ).

\section{Teneur en matière sèche}

Les données de la récolte du grain apportent un gain de précision sur l'estimation de la teneur en matière sèche équivalent à celui obtenu avec les caractères observés en cours de végétation (Tableau V). Toutefois, le gain de précision reste en général assez faible, et inférieur à celui induit par une augmentation du nombre de répétitions récoltées en fourrage (Tableau $V$ ).

\section{Recherche d'une meilleure efficacité de la sélection sur les critères grain}

\section{Rendement en grain}

Une récolte en fourrage, en complément d'une récolte en grain, peut améliorer la précision de l'estimation du rendement en grain de façon aussi importante (SRD85, SRCD85), voire plus importante (SRC84), que l'observation de caractères en cours de végétation (Tableau $V$ ). Une récolte en fourrage et en grain est plus efficace qu'un doublement du nombre de répétitions récoltées en grain (Tableau $V$ ), pour 2 populations (SRD85, SRC84). Cependant, seule l'augmentation du nombre de répétitions récoltées en grain conduirait à une meilleure estimation du rendement en grain de la population SRC85 (Tableau V).

\section{Humidité du grain}

Les caractères observés à la récolte du fourrage, tout comme les caractères notés en cours de végétation, n'améliorent pas la qualité de l'estimation de l'humidité du grain (Tableau V). L'augmentation du nombre de répétitions récoltées en grain est donc plus efficace pour améliorer l'efficacité de la sélection sur ce caractère.

\section{Conséquences au niveau des réponses à la sélection}

Les estimations multicaractères des critères fourrage et grain, tenant compte des données recueillies sur les 2 types de récolte, peuvent être combinées dans une sélection sur index. Une estimation des progrès génétiques attendus sur le rendement en fourrage et le rendement en grain par sélection sur index a été réalisée, dans le cas où les contraintes de précocité de récolte fourrage et grain devraient être simultanément respectées. Pour cet objectif de sélection très contraignant, il a été possible de maintenir des progrès génétiques sur le rendement en fourrage (ou en grain) proches de ceux attendus pour une sélection exclusivement fourrage (ou grain) sur 2 répétitions, grâce à l'efficacité des estimations multicaractères pratiquées (Tableau VI). Cet exemple montre donc l'intérêt de la prise en compte des données recueillies sur les 2 types de récolte pour l'estimation de chacun des critères de sélection, les pondérations définitives dépendant de l'objectif choisi.

\section{DISCUSSION, CONCLUSION}

Lorsque la précision des expérimentations est suffisante, la sélection directe pour un objectif de sélection, fourrage ou grain, est toujours la plus efficace. L'ampleur des réponses corrélatives est toutefois différente selon le critère de sélection. La sélection sur performances fourrage entraîne une réponse corrélative sur le rendement en grain inférieure de $20 \%$ en moyenne à la répon- 
se à la sélection grain. Elle conduit d'autre part à retenir des plantes plus tardives en récolte grain qu'une sélection grain précoce. Dans le cas d'un objectif de sélection fourrage précoce, une sélection indirecte sur performances grain induit une réponse corrélative sur le rendement en fourrage inférieure de $35 \%$ en moyenne à la réponse à une sélection directe sur performances fourrage. Une sélection sur le rendement en biomasse aérienne totale compromet donc moins le rendement en grain que la sélection grain ne limite le potentiel de production en plante entière, ce qui confirme les résultats de Gallais et al. (1981). La réponse corrélative du rendement en plante entière à la sélection grain est tout au plus égale au progrès sur le rendement en grain; une tendance à une augmentation de l'indice de récolte est donc attendue pour toutes les populations par une sélection grain. Cela est cohérent avec les résultats de Barrière et al. (1987) concernant le progrès génétique observé pour les variétés de maïs précoce. Pour un objectif de sélection grain, des progrès génétiques plus importants à terme sur le rendement en grain pourraient peut-être être atteints, en imposant, pour les premiers cycles de sélection, un progrès génétique sur la biomasse aérienne totale supérieur à la réponse corrélative induite par une sélection exclusivement grain. La sélection sur le rendement en plante entière est donc indispensable pour un objectif de sélection fourrage, mais elle apparaît aussi nécessaire pour la création de matériel à double vocation, voire spécifiquement grain. La nécessité de séparer la sélection fourrage de la sélection grain dès le début des programmes d'amélioration se trouve ainsi confirmée. Une sélection divergente fourrage et grain a d'ailleurs été amorcée pour les 4 populations étudiées, à partir des résultats expérimentaux rapportés.

Cette étude souligne également l'intérêt d'une estimation multicaractère des critères de sélection. La prise en considération de caractères observés en cours de végétation sur les descendances en croisement, ou des données recueillies à la récolte du grain, est toujours efficace pour augmenter la précision de l'estimation du rendement en fourrage. Les caractères de morphologie ou le rendement en fourrage apportent toutefois moins d'informations pour l'estimation du rendement en grain, ce qui pouvait être atten$\mathrm{du}$, du fait de la variabilité génétique existant pour les phénomènes de transiocation. Pour le blé, Brabant et al., (1989) n'observent même aucun intérêt à la mesure de caractères de morphologie pour l'estimation du rendement en grain.

Dans le cas d'une sélection exclusivement fourrage (resp. grain), une double récolte en fourrage et en grain n'est pas toujours aussi efficace que la seule augmentation du nombre de répétitions récoltées en fourrage (resp. grain), surtout lorsqu'un poids important est accordé au critère de précocité de récolte. Une sélection simultanée sur la valeur en fourrage et la valeur en grain peut cependant être nécessaire. Pour un objectif de sélection fourrage, elle peut permettre d'éliminer les génotypes peu productifs en grain. Pour la création de matériel à double vocation ou de matériel grain, l'intérêt d'une sélection portant à la fois sur la plante entière et le grain a été souligné ci-dessus. Les estimations multicaractères des critères de sélection combinant l'information apportée par les 2 types de récolte renforcent alors de façon importante l'efficacité de la sélection. Le gain de précision fourni par de telles estimations est en général équivalent à celui procuré par l'observation de caractères de morphologie. Un gain de précision supplémentaire sur les critères de valeur en croisement est encore attendu avec la prise en compte de critères de valeur propre des descendances S1 (Sampoux et al., 1989).

\section{BIBLIOGRAPHIE}

Barloy J. (1984) Biologie de l'élaboration du rendement. In: Physiologie du Maïs (A. Gallais ed.) INRA, pp. $163-180$

Barrière Y., Gallais A., Derieux M. \& Panouillé A. (1987) Etude de la valeur agronomique en plante entière au stade de récolte ensilage de différentes variétés de maïs grain sélectionnées entre 1950 et 1980. Agronomie 7 (2), 73-79

Brabant P., Manes Y., Trottet M. \& Picard E. (1989) Corrélations génétiques, héritabilités et possibilités de sélection multilocale précoce sur le rendement chez le blé tendre d'hiver. Agronomie. 9 (1), 49-54

Daynard T.B. \& Hunter R.B. (1975) Relationships among whole plant moisture, grain moisture, dry matter yield and quality of whole plant corn silage. Can J. Plant. Sci. 55, 77-84

Daynard T.B. \& Tollenaar M. (1984) Perspectives d'amélioration des maïs précoces. In: Physiologie du Maïs. (A. Gallais ed.) INRA, pp. 536-574

Deinum B. \& Struik P.C (1986) Improving the nutritive value of forage maize. In: Breeding of Silage Maize. (O. Doistra \& P. Miedema eds.) Pudoc Wageningen. pp. $77-90$

Gallais A., Vincourt P. \& Huguet L. (1981) Objectifs et critères de sélection du maïs fourrage. $X^{\theta}$ congrès de la section Eucarpia maïs-sorgho, Montreux, septembre 1981

Gallais A., Vincourt P. \& Bertholleau J.C. (1983) Etude de critères de sélection chez le maïs fourrage: héritabilités, corrélations génétiques, et réponse attendue à la sélection. Agronomie 3 (8), 751-760

Geiger H.H., Mechinger A.E. \& Schmidt G.A. (1986) Analysis of factorial crosses between flint and dent maize inbred lines for forage performance and quality 
traits. In: Breeding of Silage Maize. (O. Dolstra \& P. Miedema eds). Pudoc Wageningen. pp. 147-154

Henderson C.R. 1975) Best linear unbiased estimation and prediction under a selection model. Biometrics 21 (2), 423-447

Hunter R.B. (1986) Selecting hybrids for silage maize production: a Canadian experience. In: Breeding of Silage Maize. (O. Dolstra \& P. Miedema. eds). Pudoc Wageningen. pp. 140-146

Knapp S.J., Stroup W.W. \& Ross W.M. (1985) Exact confidence intervals for heritability on a progeny mean basis. Crop. Sci. 25, 192-194

Lila M., Barrière Y. \& Traineau R. (1986) Mise au point et étude d'un test enzymatique de la digestibilité de fourrages pauvres ou riches en amidon. Agronomie 6 (3), 285-291

Rouvier R. (1977) Mise au point sur le modèle classique d'estimation de la valeur génétique. Ann. Genét. Sel. Anim. 9, 17-26

Sampoux J.P., Gallais A. \& Lefort-Buson M. (1989) Intérêt de la valeur propre des descendances $\mathrm{S} 1$ associée à la valeur en croisement avec un testeur pour la sélection du maïs fourrage. Agronomie 9 (5), 511-520 Scheinberg E. (1966) The sampling variance of the correlation coefficients estimated in genetic experiments. Biometrics 22, 187-191

Vincourt P. \& Gallais A. (1983) Sur la recherche de critères de sélection: la régression géno-phénotypique. Agronomie 3 (9), 827-830 